\title{
Pembrolizumab plus chemotherapy for first-line treatment of metastatic nonsquamous non-small-cell lung cancer: a network meta-analysis
}

\author{
Andrew M Frederickson*,1, Stella Arndorfer ${ }^{1}$, Ina Zhang ${ }^{1}$, Maria Lorenzi ${ }^{1}$, Ralph Insinga ${ }^{2}$, \\ Ashwini Arunachalam², Thomas A Burke² \& George R Simon ${ }^{3}$ \\ ${ }^{1}$ Evidence Synthesis \& Decision Modeling, Precision Xtract, Oakland, CA 94612, USA \\ ${ }^{2}$ Center for Observational \& Real World Evidence (CORE), Merck \& Co., Inc., Kenilworth, NJ 07033, USA \\ ${ }^{3}$ Department of Thoracic/Head \& Neck Medical Oncology, The University of Texas MD Anderson Cancer Center, Houston, \\ TX 77005, USA \\ *Author for correspondence: Tel.: +1 510844 8103; andrew.frederickson@precisionxtract.com
}

\begin{abstract}
Aim: A systematic review and network meta-analysis were conducted to evaluate the efficacy of pembrolizumab + pemetrexed + platinum relative to other regimens in metastatic nonsquamous non-smallcell lung cancer (NSq-NSCLC). Patients \& methods: Eligible studies evaluated first-line regimens in NSqNSCLC patients without known targetable mutations. Relative treatment effects were synthesized with random effects proportional hazards Bayesian network meta-analyses. Results: The hazard ratio (HR) for overall survival (OS) for pembrolizumab + pemetrexed + platinum was statistically significant over all platinum-doublet (HR range: $0.42-0.61$ ), platinum-doublet + bevacizumab (HR range: $0.44-0.53$ ) and platinum-doublet + atezolizumab regimens (HR range: $0.56-0.62$ ). Additionally, pembrolizumab + pemetrexed + platinum numerically improved OS over atezolizumab + paclitaxel + carboplatin + bevacizumab (HR: $0.65 ; 95 \%$ credible interval: $0.43,1.01$ ). Pembrolizumab + pemetrexed + platinum had $95.6 \%$ probability of being the best treatment regimen for OS. Conclusion: Pembrolizumab + pemetrexed + platinum is likely the most efficacious first-line regimen for metastatic NSq-NSCLC.
\end{abstract}

First draft submitted: 1 December 2018; Accepted for publication: 11 January 2019; Published online: 4 February 2019

Keywords: immunotherapy • nonsquamous • NSCLC • PD-L1 inhibitor • pembrolizumab

The introduction of immunotherapy has led to a rapid transformation in the treatment of metastatic non-smallcell lung cancer (NSCLC). The PD-1/PD-L1 inhibitors, nivolumab, pembrolizumab and atezolizumab were first approved for advanced NSCLC in previously treated disease, based on significantly improved overall survival (OS) compared with docetaxel [1-4]. Pembrolizumab monotherapy was subsequently approved for the first-line treatment of metastatic NSCLC whose tumors have high PD-L1 expression (tumor proportion score [TPS] $\geq 50 \%$ ) and with no $E G F R$ mutation or $A L K$ gene rearrangement, based on significantly improved OS compared with platinum-based doublet chemotherapy [5].

Recently, several clinical trials evaluating PD-1/PD-L1 inhibitors in combination with traditional chemotherapy regimens have been published. In a randomized, Phase II clinical trial in metastatic nonsquamous NSCLC without actionable mutations, carboplatin plus pemetrexed with pembrolizumab provided significantly better response rates and progression-free survival (PFS) compared with chemotherapy alone, leading to FDA accelerated approval in May 2017 [6]. The subsequent confirmatory Phase III KEYNOTE-189 trial demonstrated patients who received pembrolizumab + pemetrexed + platinum had superior OS compared with patients who received pemetrexed + platinum chemotherapy alone (hazard ratio [HR] for OS: 0.49; 95\% CI: 0.38-0.64; $\mathrm{p}<0.001$ ) [7]. The Phase III IMpower150 study demonstrated carboplatin + paclitaxel + bevacizumab + atezolizumab was superior to carboplatin + paclitaxel + bevacizumab for OS (HR for OS: 0.78; 95\% CI: 0.64-0.96; $\mathrm{p}=0.0164$ ), while carboplatin + paclitaxel + atezolizumab was not superior to carboplatin + paclitaxel + bevacizumab $(\mathrm{HR}$ for 
OS: 0.88 ; 95\% CI: 0.72-1.08) [8,9]. The Phase III IMpower130 trial results add to evidence that carboplatin + nabpaclitaxel + atezolizumab has superior OS compared with carboplatin + nab-paclitaxel (HR for OS: 0.79; 95\% CI: $0.64-0.98 ; \mathrm{p}=0.033$ [10]. Finally, the Phase III IMpower132 interim analysis results failed to demonstrate that carboplatin or cisplatin + pemetrexed + atezolizumab had superior OS compared with carboplatin or cisplatin + pemetrexed (HR for OS: 0.81; 95\% CI: 0.64-1.03; p = 0.0797) [11].

KEYNOTE-021G, a randomized Phase II trial, showed benefit for platinum doublet chemotherapy in combination with immunotherapy compared with chemotherapy alone in metastatic nonsquamous NSCLC without actionable mutations. However, KEYNOTE-189 was the first randomized Phase III trial to show the benefit of adding immunotherapy to a platinum doublet chemotherapy regimen irrespective of PD-L1 expression status. On the basis of this result, the National Comprehensive Cancer Network (NCCN) issued a category 1 recommendation in its guidelines [12]. Additionally, based on findings in KEYNOTE-189, the European Society for Medical Oncology (ESMO) has issued platinum doublet chemotherapy as a category A regimen [13]. Due to KEYNOTE-189's impact on the standard of care, it was of interest to compare the relative efficacy of pembrolizumab + pemetrexed + platinum to additional competing interventions, including other immunotherapy-based regimens for the first-line treatment of metastatic nonsquamous NSCLC patients without tumor EGFR mutation or ALK translocation by means of a network meta-analysis (NMA). Given a connected network of evidence, NMA provides a valid alternative for simultaneous comparison of all included interventions and estimates relative treatment effects between any pair of interventions in the network [14-16].

\section{Methods}

\section{Systematic literature review}

A systematic literature review (SLR) was initiated in May 2016 and updated at regular intervals through November 2018. Prespecified selection criteria regarding study population, interventions, comparators, outcomes and study design (PICOS) are enumerated in Table 1. The PICOS captured randomized controlled trials (RCTs) that were conducted in metastatic NSCLC patients who were previously untreated for their disease; evaluated recommended interventions; reported outcomes of interest; were conducted after 1995; and were published in English. Trials employing PD-L1 selection criteria were not included. During each SLR update, searches of MEDLINE, EMBASE and the Cochrane Central Register of Controlled Trials were conducted. The Scottish Intercollegiate Guidelines Network (SIGN) filter for RCTs was used to limit study design. Medical subject headings (MeSH) terms and keywords were used to identify studies by population, interventions and comparators. In addition, proceedings from European Society for Medical Oncology, International Association for the Study of Lung Cancer World Conference on Lung Cancer (IASLC WCLC), American Association for Cancer Research (AACR) and the American Society of Clinical Oncology (ASCO) were searched. Full search strategies are available in the Supplementary Materials.

Two reviewers independently screened titles and abstracts of articles and conference proceedings for potentially eligible studies. Full-text publications corresponding to included studies were then retrieved and screened in duplicate. Preferred reporting items for systematic reviews and meta-analyses (PRISMA) flow diagrams for the original SLR and for each update are provided in the Supplementary Materials. Discrepancies were resolved by discussion between the pair of reviewers. Once the list of included studies was finalized, trial characteristics, patient characteristics and study results were extracted in duplicate from all eligible publications.

Study quality was assessed using Cochrane Collaboration's Risk of Bias tool to evaluate six key domains: sequence generation; allocation concealment; blinding of participants, personnel and outcome assessors; incomplete outcome data; selective outcome reporting; and other sources of bias [17]. Specific study characteristics evaluated include: random sequence generation, allocation concealment, blinding/masking status, incomplete outcome data, selective outcome reporting, as well as funding, support or conflicts of interest reported. Each of the study characteristics is assigned a low, medium, high or unclear risk status, and support for the decision is made and reported in tables (Supplementary Information).

\section{Network meta-analysis}

NMA is a well-established statistical technique that allows the comparison of treatment effects among interventions that have not been studied in a head-to-head clinical trial $[14-16,18]$. Indirect treatment comparisons have been in use for $>20$ years and a recent review found that nearly 800 NMAs were published in over 300 journals between 1997 and 2015 [19,20]. Additionally, health technology assessment bodies in a number of countries recommend Bayesian NMA to synthesize evidence for decision making [18,21]. The methods followed in this analysis match 
Table 1. Study selection criteria to identify trials for the systematic literature review.

\begin{tabular}{|c|c|}
\hline Criteria & Description \\
\hline Population & Metastatic NSCLC patients who were previously untreated with systemic therapy for their metastatic nonsquamous disease ${ }^{\dagger}$ \\
\hline \multirow[t]{29}{*}{ Interventions } & Pembrolizumab \\
\hline & Pembrolizumab + platinum + pemetrexed \\
\hline & Nivolumab \\
\hline & Nivolumab + ipilimumab \\
\hline & Nivolumab + platinum (carboplatin + cisplatin) + pemetrexed, followed by pemetrexed maintenance \\
\hline & Durvalumab \\
\hline & Durvalumab + tremelimumab \\
\hline & Atezolizumab \\
\hline & Atezolizumab + bevacizumab + paclitaxel + carboplatin \\
\hline & Atezolizumab + paclitaxel + carboplatin \\
\hline & Atezolizumab + nab-paclitaxel + carboplatin \\
\hline & Atezolizumab + platinum + pemetrexed \\
\hline & Avelumab \\
\hline & Pemetrexed + platinum \\
\hline & Pemetrexed + platinum, followed by pemetrexed maintenance \\
\hline & Taxane + platinum (paclitaxel, nab-paclitaxel or docetaxel) \\
\hline & Docetaxel + platinum followed by pemetrexed maintenance \\
\hline & Paclitaxel + platinum followed by pemetrexed maintenance \\
\hline & Gemcitabine + platinum \\
\hline & Gemcitabine + platinum followed by pemetrexed maintenance \\
\hline & Vinorelbine + platinum \\
\hline & Gemcitabine + platinum followed by gemcitabine maintenance \\
\hline & Etoposide + platinum \\
\hline & Nonpemetrexed platinum doublet followed by pemetrexed switch maintenance \\
\hline & Nonerlotinib-containing platinum doublet $\times$ 4-6 cycles followed by erlotinib switch maintenance \\
\hline & Gemcitabine + bevacizumab + platinum \\
\hline & Paclitaxel + bevacizumab + platinum \\
\hline & Pemetrexed + bevacizumab + platinum \\
\hline & Nonplatinum regimens (gemcitabine + docetaxel and gemcitabine + vinorelbine) \\
\hline \multirow[t]{2}{*}{ Comparisons } & Any of the above interventions and placebo \\
\hline & Between any of the above interventions \\
\hline \multirow[t]{5}{*}{ Outcomes } & Overall survival \\
\hline & Progression-free survival \\
\hline & Overall response rate (tabulations only) \\
\hline & Health-related quality of life (tabulations only) \\
\hline & Grade 3 or 4 adverse events (tabulations only) \\
\hline Study design & Randomized controlled trials \\
\hline \multirow[t]{2}{*}{ Other } & English language \\
\hline & 1995 onwards \\
\hline \multicolumn{2}{|c|}{$\begin{array}{l}\text { †The population of interest is metastatic nonsquamous NS } \\
\text { anticipated lack of trial data for this specific population. } \\
\text { Nota bene: Platinum is carboplatin or cisplatin. } \\
\text { NSCLC: Non-small-cell lung cancer. }\end{array}$} \\
\hline
\end{tabular}

those outlined in the Technical Support Document \#2, published by the National Institute for Health and Care Excellence in the UK. In brief, NMA is an extension of pairwise meta-analysis that allows estimation of relative treatment effects between all interventions in a connected network of RCTs by calculating the differences in pooled relative treatment effects between studies. First, a parameter such as an HR is assumed to follow a noninformative (i.e., no prior knowledge of the parameter) probability distribution. Next, the probabilities of different values for the parameters of interest are calculated given the observed data, and the prior probability distribution is updated to yield a posterior probability distribution. This calculation is performed using a Markov chain Monte Carlo 
algorithm implemented in the software package OpenBUGS according to the methods described in National Institute for Health and Care Excellence Technical Support Document \#2 [18]. The posterior distribution is then summarized as a point estimate for the parameter of interest along with a $95 \%$ credible interval (CrI). CrIs are the Bayesian analog of a frequentist CI. Unlike CIs, CrIs are interpreted probabilistically; there is $95 \%$ chance that the true value of the parameter lies within the $95 \% \mathrm{CrI}$.

A well-designed NMA is dependent upon properly defining both prognostic factors and relative treatment effect modifiers. Prognostic factors are characteristics that affect the outcome of patients with a disease, independent of treatment. Relative treatment effect modifiers are patient characteristics that influence the treatment effects of an intervention relative to the control, and which may introduce between-study heterogeneity if effect modifiers are imbalanced across trials. Only the relative treatment effects of each trial are considered, reducing the degree to which indirect comparisons of interventions are affected by differences in study effects (i.e., known and unknown prognostic factors). Therefore, in order to decrease the likelihood of biased estimates, potential relative treatment effect modifiers must be evaluated in a feasibility assessment prior to conducting an NMA to ensure included trials are reasonably similar.

Before proceeding with an NMA, a feasibility assessment was conducted to determine whether the RCT evidence for the interventions of interest form a single evidence network for each population and outcome of interest; and assess distributions of treatment, outcomes, study and patient characteristics that may influence the treatment effects when performing direct comparisons of the evidence networks [22]. Assessed trial characteristics included study phase, trial initiation, trial completion, trial region(s), eligibility criteria and risk of bias. Heterogeneity of patient characteristics was determined by baseline patient characteristics such as crossover, demographics of the trial (e.g., age, sex, race/ethnicity) and clinical characteristics of the trial (e.g., ECOG performance status, smoking status and disease stage). Histology, a known treatment effect modifier, was accounted for by including only patients with nonsquamous disease in the analysis, as stated in the PICOS eligibility criteria. Trials that enrolled patients of other histologies were included only if outcomes were available for the nonsquamous subgroup and these subgroup outcomes were used in the analysis. After determining that the evidence networks and their respective trials were sufficiently similar, the evidence was synthesized by means of NMAs. Random-effects pairwise meta-analyses were used to determine direct and indirect associated treatment effects for both OS and PFS NMAs. Under the assumption of consistency, the NMA model relates the data from the individual studies to basic parameters reflecting the (pooled) relative treatment effect of each intervention compared with the reference, in this case platinum + pemetrexed. Based on these basic parameters, the relative treatment effects between each of the contrasts in the network were obtained. Under the Bayesian framework, it is possible to calculate the probability of being the best treatment out of all treatments included in the connected network of evidence. It is also possible to calculate which treatments are the second best, third best, etc., treatment out of all interventions included in the connected network [23].

The NMA of reported HRs for PFS and OS, assuming proportional hazards between treatments, was performed using a model with a contrast-based normal likelihood for the log HR (and corresponding standard error) of each trial (or comparison) in the network. Normal noninformative prior distributions for the parameters were estimated with a mean of 0 and a variance of 10,000. In cases where HRs were not reported but Kaplan-Meier curves were available, the Kaplan-Meier curves were digitized (DigitizeIt; www.digitizeit.de/) and constant HRs were estimated using the Guyot algorithm [24].

The results of the NMA for PFS and OS were presented with estimates for treatment effects of each intervention relative to the reference, platinum + pemetrexed. The posterior distributions of relative treatment effects were summarized by the median and $95 \%$ CrIs, which were constructed from the 2.5 th and 97.5 th percentiles of the posterior distributions. Additionally, median rankings and associated probability of being the best treatment for each outcome of interest were presented. All analyses were performed using R version 3.4.0 (R Project for Statistical Computing) with the package R2JAGS version 0.5.7 (OpenBUGS Project Management Group) [25]. $\mathrm{R}$ is a widely used, free, open-source programming language and environment for statistical computing as well as graphics [25].

\section{Results}

Systematic literature review \& feasibility assessment

The original SLR was conducted in May 2016 and updated in March 2017; November 2017; April 2018; June 2018; and November 2018. A total of 14,300 abstracts and 462 full text publications were screened. In all, 65 
publications pertaining to 27 trials matching the PICOS criteria were identified. The included trials and their corresponding publications are listed in Table 2.

\section{Outcomes of interest}

26 trials reported OS and 23 trials reported PFS. Eleven trials defined OS; of these trials, six defined OS as time from randomization to death from any cause. Two trials, Spigel (2012) and Sun (2015), defined OS as time from enrollment to time of death [81,82]. TRAIL defined OS as time from the first date of treatment to time of death from any cause []. Finally, both IMpower130 and IMpower132 defined OS as from baseline until death [10,11]. PFS was defined in 12 trials, of which eight trials defined PFS as time from randomization to the first documented disease progression or death. Sun (2015) defined PFS as time from enrollment to disease progression or death, and TRAIL defined time to progression (TTP) as time from first date of treatment until disease progression or death [82]. Additionally, both IMpower130 and IMpower132 defined PFS as baseline until disease progression or death, whichever occurred first $[10,11]$. Two trials reported TTP only, Checkmate 026 and TRAIL; this end point was used as a surrogate for PFS [31]. Additionally, Zhang (2013), Gronberg (2009), and Johnson (2004) reported neither PFS nor TTP and, therefore, were not included in PFS analyses [40,84,85]. One trial did not report any outcomes of interest; Kristensen (2017), reported only patient-reported outcomes [74].

\section{Trial characteristics}

Trial characteristics of included RCTs were similar (Table 3). The earliest study was completed in 2007 and the latest study is scheduled to be completed in 2019. Most trials were open label, had low risk of bias according to the Cochrane Collaboration's tool, and included patients with ECOG performance scores of 0 or 1 . However, five trials did permit patients with ECOG scores of 2 (Gronberg (2009), Jang (2017), Johnson (2004), RodriguesPereira (2011) and TRAIL) [40,50,80,85]. Based on eligibility criteria defined in the PICOS table (Table 2), only patients with nonsquamous histology were of interest. However, six trials were conducted in both nonsquamous and squamous patients. For trials including both nonsquamous and squamous patients, subgroup data in nonsquamous patients was used to maintain a homogeneous population with respect to histology status. Two trials restricted eligibility based on PD-L1 immunohistochemistry status, KEYNOTE-024 and CheckMate-026, which allowed only PD-L1-expressing patients [5,30].

\section{Intervention characteristics}

Intervention characteristics of included trials, including dosage and frequency, and planned duration of treatment were reasonably similar across trials. Treatment regimens largely consisted of six planned cycles with each cycle lasting 21 days. However, 17 trials did allow maintenance therapies for patients with complete or partial tumor response, with no progressive disease, and/or at the local physicians' discretion. Maintenance therapies consisted of certain components of the randomized regimen a patient was assigned to. Seven trials allowed for within-trial crossover in treatment following disease progression: CheckMate 026, IMpower130, Johnson 2004, KEYNOTE189, KEYNOTE-021G, KEYNOTE-024, and TRAIL [5-7,10,30,66,85]. Crossover proportions refer to patients who were eligible and crossed over to interventions upon verified disease progression (Table 5). Of trials permitting crossover, six out of the seven allowed patients to crossover from the control arm to the crossover therapy. Proportions of patients crossing over from the control arm to the maintenance therapy ranged from 40.8 to $59.4 \%$. The TRAIL trial permitted patients in both the control and intervention arms to crossover; in the control arm, $72.7 \%$ crossed over and the comparator arm had 69.0\% crossed over []. One trial evaluated nivolumab monotherapy in PD-L1positive patients, CheckMate 026 [30].

\section{Patient characteristics}

Differences identified in the ITT populations with respect to age, sex, ECOG status, smoking status and American Joint Committee on Cancer (AJCC) stage status were assessed and deemed reasonably similar. However, substantial variation was observed for ethnicity (Table 4).

Ranging from 54 to 77 years, median age at baseline varied little between treatment arms. However, two trials were conducted in exclusively elderly patients, Jang 2017 and Spigel (2012) [50,81]. Similarly, sex distribution across trials had little variation, with all trials being majority male, except for KEYNOTE-021G, which consisted of 39.0\% males [6]. Although the between-trial sex distribution was reasonably similar, within-trial sex distributions for three trials were imbalanced. Fewer than half (48\%) of the patients in the docetaxel + platinum arm of Rodrigues-Pereira 
Systematic Review Frederickson, Arndorfer, Zhang et al.

Table 2. List of publications included after full-text review, arranged by trial.

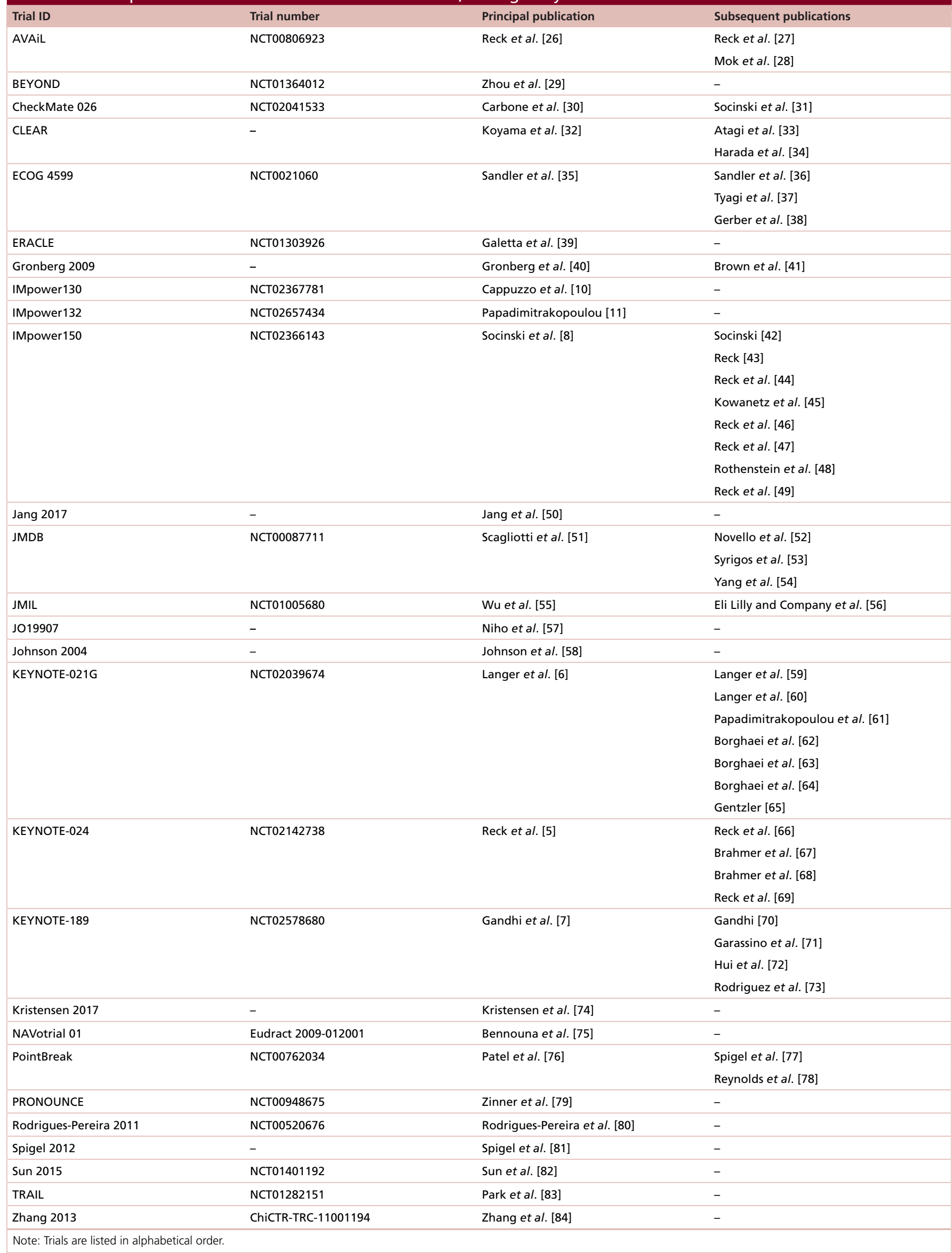




\begin{tabular}{|c|c|c|c|c|c|c|}
\hline Trial ID & Phase & Masking & Multicenter & Age (years) & Disease stage & Performance score \\
\hline AVAiL & III & Double blind & Yes & $\geq 18$ & IIIB or IV & $\mathrm{ECOG} \leq 1$ \\
\hline CheckMate 026 & III & Open label & Yes & $\geq 18$ & IV & $E C O G \leq 1$ \\
\hline CLEAR & ॥ & Open label & Yes & $20-74$ & IIIB or IV & $E C O G \leq 1$ \\
\hline ECOG 4599 & III & Open label & - & - & IIIB or IV & $\mathrm{ECOG} \leq 1$ \\
\hline Gronberg 2009 & III & Open label & Yes & $\geq 18$ & IIIB or IV & $E C O G \leq 2$ \\
\hline IMpower130 & III & Open label & Yes & $\geq 18$ & IV & $\mathrm{ECOG} \leq 1$ \\
\hline IMpower132 & III & Open label & Yes & $\geq 18$ & IV & $\mathrm{ECOG} \leq 1$ \\
\hline IMpower150 & III & Open label & Yes & $\geq 18$ & IV & $\mathrm{ECOG} \leq 1$ \\
\hline Jang 2017 & $\|$ & - & Yes & $\geq 65$ & IIIB or IV & $\mathrm{ECOG} \leq 2$ \\
\hline Johnson 2004 & ॥ & - & Yes & $\geq 18$ & IIIB or IV & $\mathrm{ECOG} \leq 2$ \\
\hline KEYNOTE-021G & ॥ & Open label & Yes & $\geq 18$ & IIIB or IV & $\mathrm{ECOG} \leq 1$ \\
\hline KEYNOTE-024 & II & Open label & Yes & $\geq 18$ & IV & $E C O G \leq 1$ \\
\hline KEYNOTE-189 & III & $\begin{array}{l}\text { Quadruple or double } \\
\text { blind }\end{array}$ & Yes & $\geq 18$ & IV & $E C O G \leq 1$ \\
\hline Kristensen 2017 & - & Open label & No & - & IIIB or IV & WHO 0-2 \\
\hline NAVotrial 01 & II & - & Yes & $18-75$ years & IIIB or IV & Karnofsky $\geq 80 \%$ \\
\hline PointBreak & III & Open label & Yes & $\geq 18$ & IIIB or IV & $E C O G \leq 1$ \\
\hline PRONOUNCE & III & Open label & Yes & $\geq 18$ & IV & $\mathrm{ECOG} \leq 1$ \\
\hline $\begin{array}{l}\text { Rodrigues-Pereira } \\
2011\end{array}$ & III & Open label & Yes & $\geq 18$ & IIIB or IV & $\mathrm{ECOG} \leq 2$ \\
\hline
\end{tabular}

(2011) were male but 54.3\% of the patients in the entire trial were male [80]. In Spigel (2012), 47\% of the patients in the pemetrexed + bevacizumab + platinum arm were male; however, $50 \%$ of the entire trial was male [81]. Similarly, fewer than half $(46 \%)$ of the patients in the paclitaxel + bevacizumab $(15 \mathrm{mg} / \mathrm{kg})+$ platinum arm of Johnson (2004) were male; however, $60 \%$ of the patients in the entire trial were male [85]. Most patients had ECOG performance status (PS) of 0 or 1. Three trials (Gronberg [2009], Rodrigues-Pereira [2011] and TRAIL) reported at least $10 \%$ of patients with an ECOG PS of 2 [40,80]. Among trials reporting this information, most $(>50 \%)$ patients were current and former smokers with the exception of TRAIL, in which slightly fewer than half of patients (48.6\%) were current and former smokers []. The proportion of patients with AJCC stage IV lung cancer was reasonably similar between all arms, ranging from 62 to $100 \%$. A minority of patients in some trials had stage IIIB disease, with KEYNOTE-189 containing no stage IIIB patients and Jang 2017 having the highest proportion of stage IIIB patients (31.5\%) [7,84]. Although many trials did not report EGFR mutation or $A L K$ translocation status, six trials were conducted in $E G F R$ mutation-negative patients only and five trials were conducted in $A L K$ translocation-negative patients only. Three trials reported minimal proportions of patients whose tumors were EGFR mutation positive ranging from 10 to $28 \%$. IMpower150, BEYOND and CLEAR reported $<8 \%$ of patients with $A L K$ translocation positive status. Although IMpower150 included some $E G F R$ and $A L K$-positive patients, HRs from the EGFR wild-type subgroup were used in the NMAs. Ethnicity varied between trials as six trials (BEYOND; JO19907; JMIL; Sun [ 2015]; Zhang [2013]; and TRAIL) were conducted among exclusively east Asian patients while seven trials (CheckMate 026, IMpower130, JMDB, KEYNOTE-021G, 


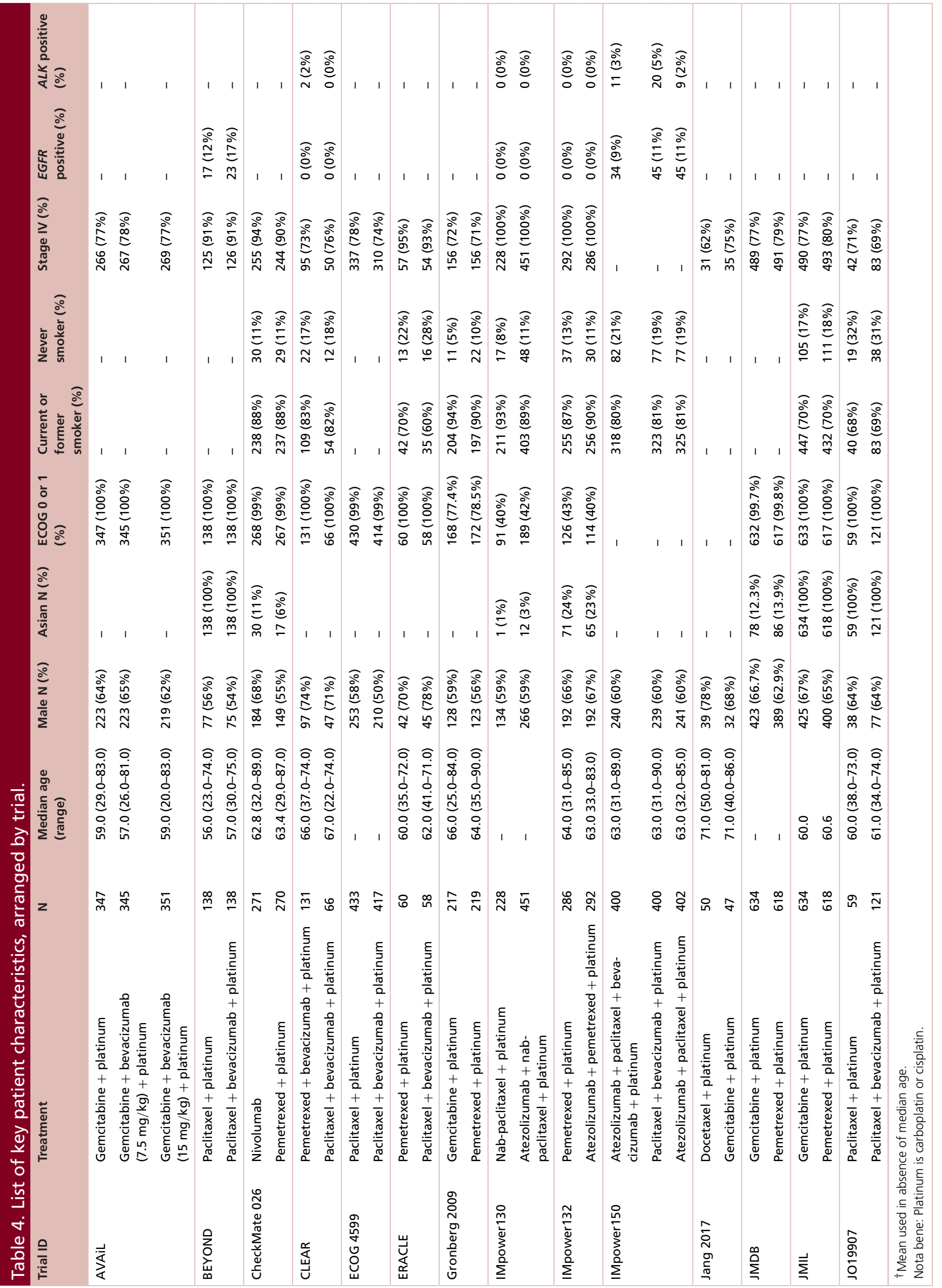




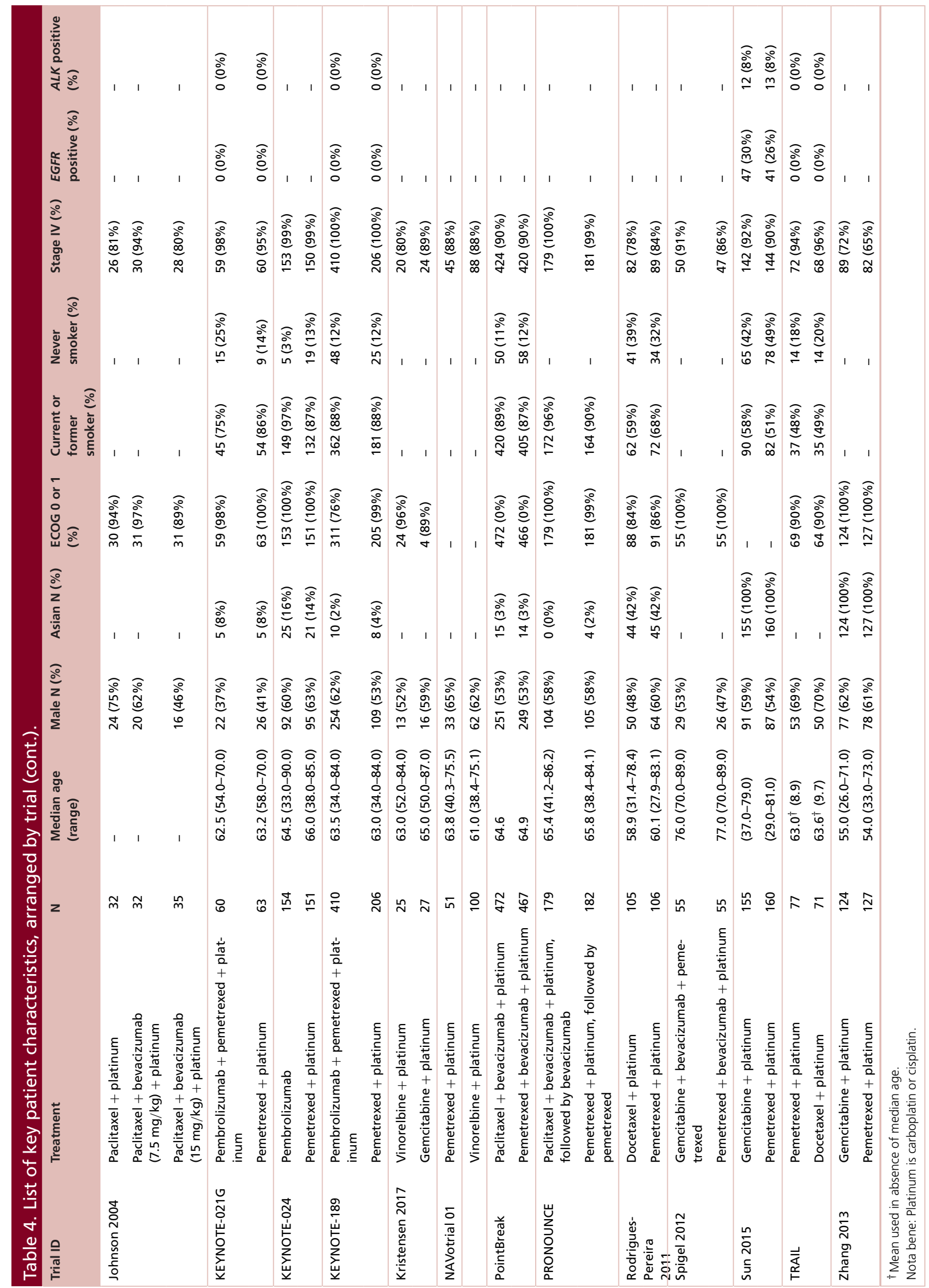




\begin{tabular}{|c|c|c|c|c|c|c|}
\hline Trial & Original treatment(s) & $\mathbf{N}$ & $\begin{array}{l}\text { Crossover } \\
\text { allowed }\end{array}$ & Crossover treatment & $\begin{array}{l}\text { Conditions for } \\
\text { crossover }\end{array}$ & $\begin{array}{l}\% \text { patients } \\
\text { crossover }\end{array}$ \\
\hline \multirow[t]{2}{*}{ CheckMate 026} & Pemetrexed + platinum & 270 & Yes & Nivolumab & Disease progression & $58.0 \%$ \\
\hline & Nivolumab & 271 & - & - & - & - \\
\hline \multirow[t]{2}{*}{ IMpower130 } & Nab-paclitaxel + platinum & 228 & Yes & $\begin{array}{l}\text { Atezolizumab + } \\
\text { nab-paclitaxel + platinum }\end{array}$ & Disease progression & $40.8 \%$ \\
\hline & Atezolizumab + nab-paclitaxel + platinum & 451 & - & - & - & - \\
\hline \multirow[t]{3}{*}{ Johnson, 2004} & Paclitaxel + platinum & 32 & Yes & Bevacizumab & Disease progression & $59.4 \%$ \\
\hline & Paclitaxel + bevacizumab $(7.5 \mathrm{mg} / \mathrm{kg})+$ platinum & 32 & - & - & - & - \\
\hline & Platinum + paclitaxel + bevacizumab $(15 \mathrm{mg} / \mathrm{kg})$ & 35 & - & - & - & - \\
\hline \multirow[t]{2}{*}{ KEYNOTE-021G } & Pemetrexed + platinum & 63 & Yes & Pembrolizumab & Disease progression & $43.3 \%$ \\
\hline & Pembrolizumab + pemetrexed + platinum & 60 & - & - & - & \\
\hline \multirow[t]{2}{*}{ KEYNOTE-024 } & Pemetrexed + platinum & 151 & Yes & Pembrolizumab & Disease progression & $43.7 \%$ \\
\hline & Pembrolizumab & 154 & - & - & - & - \\
\hline \multirow[t]{2}{*}{ KEYNOTE-189 } & Pemetrexed + platinum & 206 & Yes & Pembrolizumab & Disease progression & $41.3 \%$ \\
\hline & Pembrolizumab + pemetrexed + platinum & 410 & - & - & - & - \\
\hline \multirow[t]{2}{*}{ TRAIL } & Pemetrexed + platinum & 77 & Yes & Docetaxel or EGFR inhibitor & Disease progression & $72.7 \%$ \\
\hline & Docetaxel + platinum & 71 & Yes & Pemetrexed or EGFR inhibitor & Disease progression & $69.0 \%$ \\
\hline
\end{tabular}

KEYNOTE-189, PointBreak and PRONOUNCE) reported that fewer than 15\% of patients were of Asian/east Asian descent $[6,7,29,31,51,55,57,76,79,82,84]$.

After evaluating heterogeneity of trials included after the SLR in the feasibility assessment, five trials were excluded from NMAs. Two trials were conducted in an exclusively elderly population, Jang (2017) and Spigel (2017) [50,81]. One trial, Kristensen (2017), did not report any outcomes of interest [74]. KEYNOTE-024 and CheckMate 026 only enrolled PD-L1-expressing patients, and were thereby excluded, as no other trials in the network had this criterion and PD-L1 expression is a known effect modifier [30,66]. After exclusion of these five trials, studies were determined to be of good quality and with minimal heterogeneity, although key differences were identified with respect to within-trial crossover, use of maintenance therapies and race/ethnicity. Of trials included in the evidence base that reported subgroup analyses to assess potential effect modifiers, there was no reporting of statistically significant differences in efficacy by subgroup; however, it should be noted that in some instances potential differences by subgroup were reported numerically, but not statistically tested. In absence of individual patient data to adjust for differences identified, there is risk of confounding bias if these differences act as treatment effect modifiers. Thus, differences between the target population and the evidence base should be acknowledged when interpreting results of the NMAs conducted for OS and PFS. After the feasibility assessment, connected networks of evidence were constructed for OS and PFS consisting of 22 trials (Figure 1) and 19 trials (Figure 2), respectively.

\section{Network meta-analysis}

For each trial in the OS and PFS networks, the HR, $\log (\mathrm{HR})$ and associated standard error are presented in Tables 6 and 8, respectively. Results of the random-effects NMA for OS and PFS are shown in Tables 7 and 9, respectively.

Under the proportional hazards random-effects model, the point estimate of the HR for OS for pembrolizumab + pemetrexed + platinum was statistically significant over all platinum-based doublets (HR range: 0.42-0.61), platinum doublet + bevacizumab regimens (HR range: $0.44-0.53$ ) and both three-drug atezolizumab regimens (HR range: 0.56-0.62). Pembrolizumab + pemetrexed + platinum showed numerical superiority over the four-drug atezolizumab regimen for OS (HR: 0.65; 95\% CrI: 0.43, 1.01). However, this difference was not statistically significant. Platinum doublet regimens were statistically equivalent to each other for OS; paclitaxel + bevacizumab + platinum, as well as all atezolizumab-containing regimens were statistically superior to paclitaxel/nab-paclitaxel + platinum. Median ranks of all interventions included in the NMA for OS are provided in Table 10. Pembrolizumab + pemetrexed + platinum is $95.6 \%$ most likely to be the best treatment for OS. All treatments were $<1 \%$ likely to be the best treatment, excluding the four-drug atezolizumab regimen, which had a median rank of the second best treatment and $2.6 \%$ probability of being the best treatment for OS. 


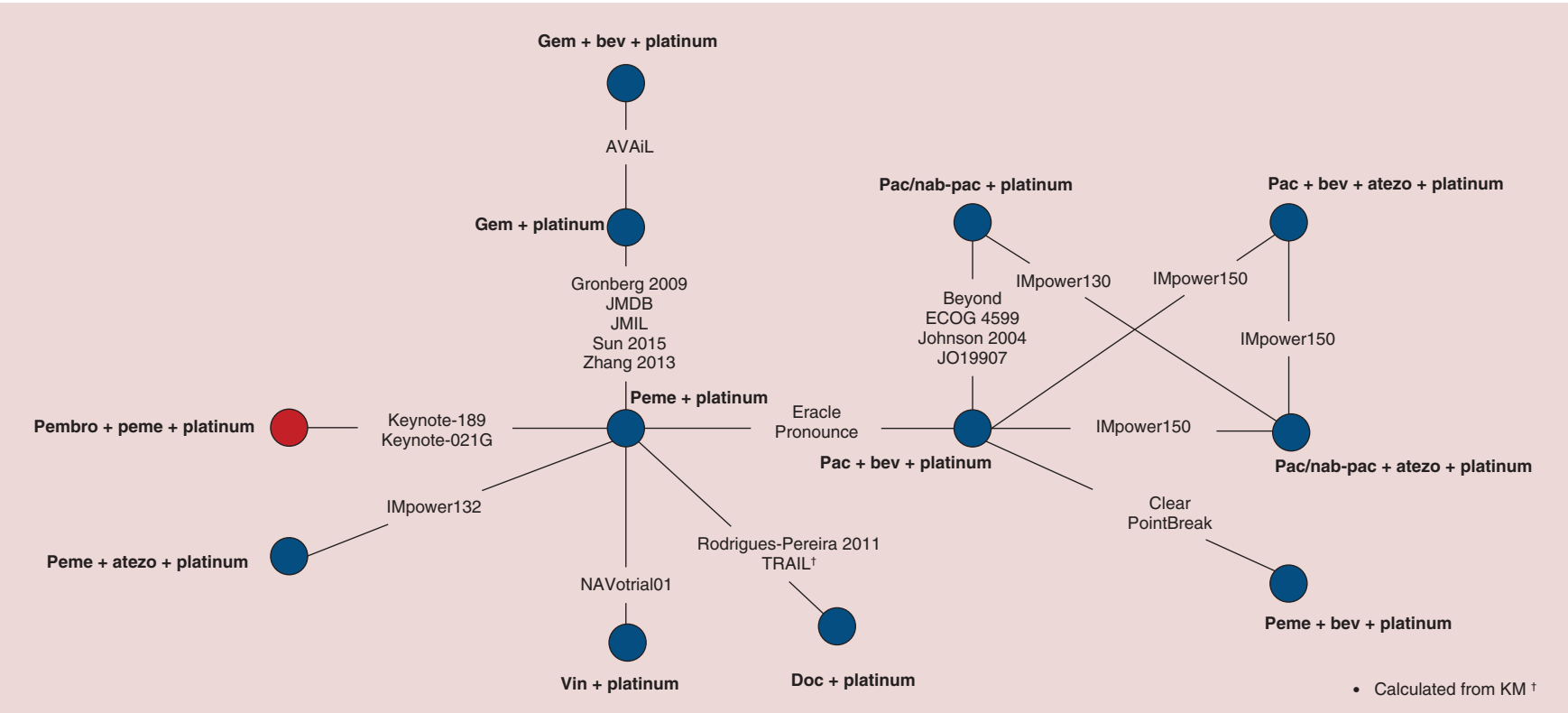

Note: atezo - atezolizumab, bev - bevacizumab, doc - docetaxel, gem - gemcitabine, pac - paclitaxel, peme - pemetrexed, pembro - pembrolizumab, platinum - carboplatin or cisplatin, nab-pac - nab-paclitaxel, vin - vinorelbine

Figure 1. Network of evidence for overall survival.

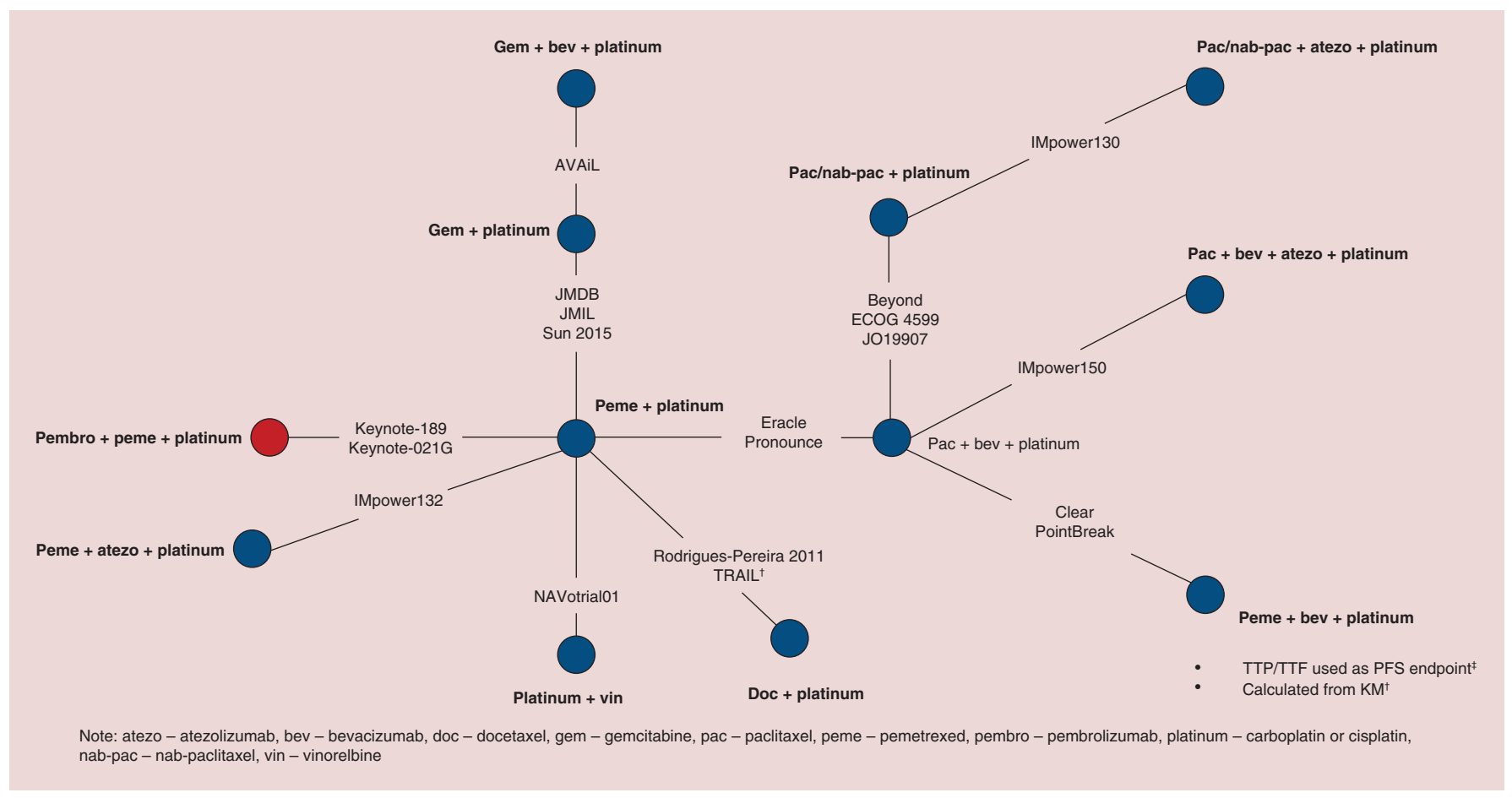

Figure 2. Network of evidence for progression-free survival.

Pembrolizumab + pemetrexed + platinum was numerically superior to all competing interventions for PFS and this difference was statistically significant for all treatments except pemetrexed + bevacizumab + platinum (HR: 0.61; 95\% CrI: 0.36, 1.01), pemetrexed + atezolizumab + platinum (HR: 0.69; 95\% CrI: 0.42, 1.14) and paclitaxel + bevacizumab + atezolizumab + platinum (HR: 0.85; 95\% CrI: 0.47, 1.48). For PFS, all interventions included in the network were superior to paclitaxel/nab-paclitaxel + platinum, with the exception 


\begin{tabular}{|c|c|c|c|c|}
\hline Study & Reference & Intervention & HR & $\log \mathrm{HR}(\mathrm{SE})$ \\
\hline AVAiL & Gem + platinum & Gem + bev + platinum & 1.03 & $0.03(0.09)$ \\
\hline BEYOND & $\mathrm{Pac} /$ nab-pac + platinum & $\mathrm{Pac}+\mathrm{bev}+$ platinum & 0.68 & $-0.39(0.16)$ \\
\hline CLEAR & $\mathrm{Pac}+\mathrm{bev}+$ platinum & Peme + bev + platinum & 0.96 & $-0.05(0.22)$ \\
\hline ECOG 4599 & Pac/nab-pac + platinum & $\mathrm{Pac}+$ bev + platinum & 0.79 & $-0.24(0.08)$ \\
\hline ERACLE & Peme + platinum & $\mathrm{Pac}+$ bev + platinum & 1.08 & $0.08(0.22)$ \\
\hline Gronberg 2009 & Peme + platinum & Gem + platinum & 1.04 & $0.04(0.13)$ \\
\hline IMpower130 & Pac/nab-pac + platinum & Pac/nab-pac + atezo + platinum & 0.79 & $-0.24(0.11)$ \\
\hline IMpower132 & Peme + platinum & Peme + atezo + platinum & 0.81 & $-0.21(0.12)$ \\
\hline IMpower150 & Pac/nab-pac + platinum & Pac/nab-pac + atezo + platinum & 0.88 & $-0.13(0.10)$ \\
\hline IMpower150 & Pac/nab-pac + platinum & $\mathrm{Pac}+\mathrm{bev}+$ atezo + platinum & 0.78 & $-0.25(0.10)$ \\
\hline J019907 & $\mathrm{Pac} /$ nab-pac + platinum & $\mathrm{Pac}+$ bev + platinum & 0.99 & $-0.01(0.21)$ \\
\hline JMDB & Peme + platinum & Gem + platinum & 1.23 & $0.21(0.08)$ \\
\hline JMIL & Peme + platinum & Gem + platinum & 1.00 & $0.00(0.15)$ \\
\hline Johnson 2004 & Pac/nab-pac + platinum & $\mathrm{Pac}+\mathrm{bev}+$ platinum & 0.85 & $-0.16(0.27)$ \\
\hline KEYNOTE-21G & Peme + platinum & Pembro + peme + platinum & 0.56 & $-0.58(0.28)$ \\
\hline KEYNOTE-189 & Peme + platinum & Pembro + peme + platinum & 0.49 & $-0.71(0.13)$ \\
\hline NAVotrial01 & Peme + platinum & Vin + platinum & 1.00 & $0.00(0.22)$ \\
\hline PointBreak & $\mathrm{Pac}+\mathrm{bev}+$ platinum & Peme + bev + platinum & 1.00 & $0.00(0.08)$ \\
\hline PRONOUNCE & Peme + platinum & $\mathrm{Pac}+\mathrm{bev}+$ platinum & 0.93 & $-0.07(0.13)$ \\
\hline Rodrigues-Pereira (2011) & Peme + platinum & Doc + platinum & 1.01 & $0.01(0.17)$ \\
\hline Sun (2015) & Peme + platinum & Gem + platinum & 1.14 & $0.13(0.17)$ \\
\hline TRAIL & Peme + platinum & Doc + platinum & 0.68 & $-0.39(0.18)$ \\
\hline Zhang (2013) & Peme + platinum & Gem + platinum & 1.05 & $0.05(0.17)$ \\
\hline
\end{tabular}

Atezo: Atezolizumab; Bev: Bevacizumab; Doc: Docetaxel; Gem: Gemcitabine; HR: Hazard ratio; Pac: Paclitaxel; Pembro: Pembrolizumab; Peme: Pemetrexed; SE: Standard error; Vin: Vinorelbine.

of vinorelbine + platinum (HR: 0.64; 95\% CrI: 0.33, 1.16). Median ranks for interventions included in the NMA for PFS are provided in Table 11. Pembrolizumab + pemetrexed + platinum had a median rank of being the best treatment regimen, and was associated with a $67.3 \%$ of being the best treatment. Following pembrolizumab + pemetrexed + platinum, the four-drug atezolizumab regimen was associated with a median rank of second best, and $24.1 \%$ likely to be the best treatment regimen. All other treatment regimens had $<5 \%$ likelihood of being the best treatment regimen for PFS.

\section{Discussion}

The objective of this study was to assess the efficacy of pembrolizumab + pemetrexed + platinum relative to alternative interventions for first-line treatment of metastatic NSCLC patients whose tumors had nonsquamous histology and are sensitizing EGFR mutation and $A L K$ translocation negative. The validity of an NMA depends on the quality of the RCTs and the extent of any violations in the similarity and consistency assumptions across studies. In an NMA of RCTs involving multiple treatment comparisons, randomization holds only within the individual trials and not across trials. If the different direct comparisons show systematic differences in study and patient characteristics, and these differences are treatment-effect modifiers, then the estimates of any indirect comparison as obtained with the NMA will be biased. To assess these risks, a feasibility assessment examining heterogeneity in terms of treatment and outcome characteristics, as well as the study and patient characteristics, was performed [22]. Three trials were excluded from analyses as they differed from the rest of evidence base with respect to age and eligibility criteria. Furthermore, one trial was removed as it only reported patient-reported outcomes and another trial evaluated a treatment that is not approved for the first-line treatment of nonsquamous NSCLC patients. After removal of these five trials, the trials included in the NMAs were largely similar in trial and patient characteristics. It is important to recognize that only trials meeting the prespecified PICOS eligibility criteria and feasibility assessment criteria were included. Extrapolation of results to populations other than nonsquamous NSCLC patients with PS $0-1$ and without targetable mutations would not be valid. 


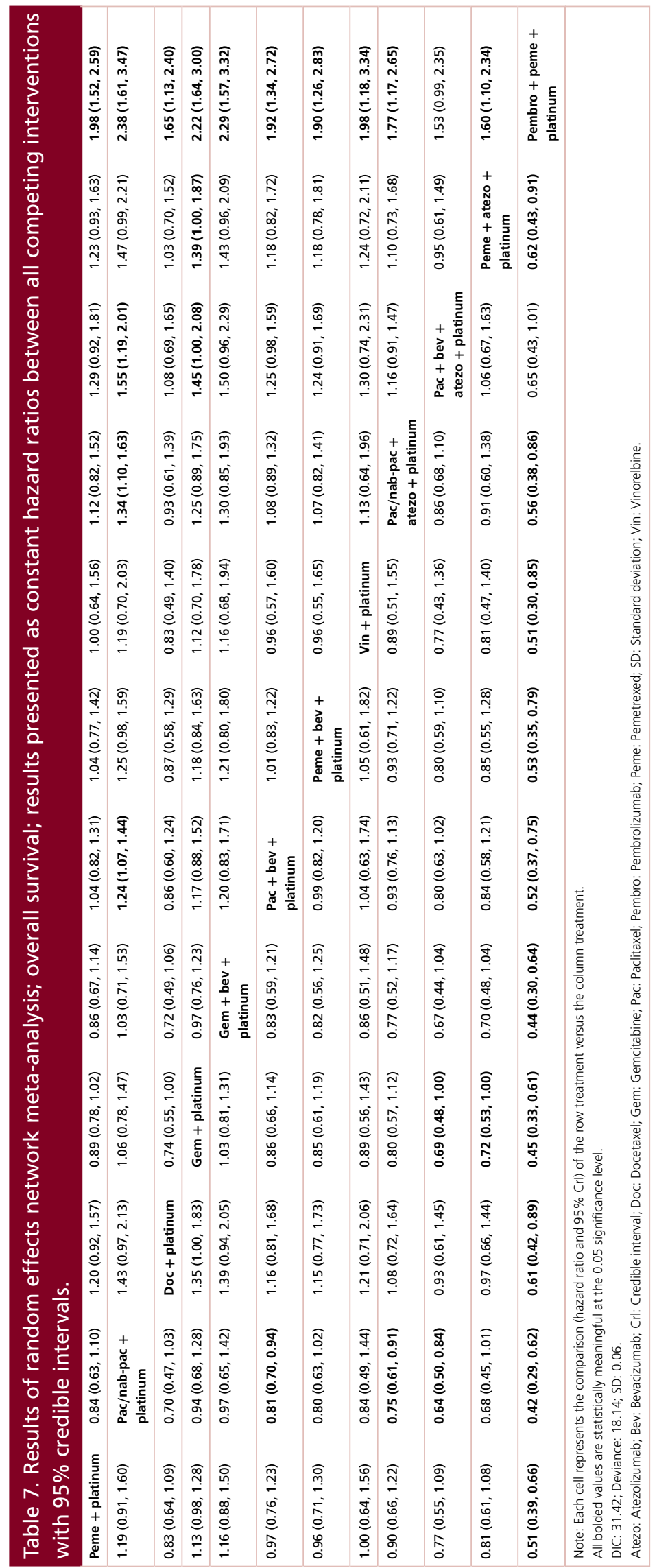




\begin{tabular}{|c|c|c|c|c|}
\hline Study & Reference & Intervention & HR & $\log H R(S E)$ \\
\hline AVAiL & Gem + platinum & Gem + bev + platinum & 0.82 & $-0.20(0.09)$ \\
\hline BEYOND & $\mathrm{Pac} / \mathrm{nab}-\mathrm{pac}+$ platinum & $\mathrm{Pac}+\mathrm{bev}+$ platinum & 0.40 & $-0.92(0.16)$ \\
\hline CLEAR & $\mathrm{Pac}+$ bev + platinum & Peme + bev + platinum & 0.83 & $-0.19(0.16)$ \\
\hline ECOG 4599 & Pac/nab-pac + platinum & $\mathrm{Pac}+$ bev + platinum & 0.66 & $-0.42(0.08)$ \\
\hline ERACLE & Peme + platinum & $\mathrm{Pac}+$ bev + platinum & 1.27 & $0.24(0.20)$ \\
\hline IMpower130 & Pac/nab-pac + platinum & $\mathrm{Pac} / \mathrm{nab}-\mathrm{pac}+$ atezo + platinum & 0.64 & $-0.45(0.09)$ \\
\hline IMpower132 & Peme + platinum & Peme + atezo + platinum & 0.76 & $-0.28(0.10)$ \\
\hline IMpower150 & $\mathrm{Pac} / \mathrm{nab}-\mathrm{pac}+$ bev + platinum & Pac + bev + atezo + platinum & 0.59 & $-0.53(0.09)$ \\
\hline JMDB & Peme + platinum & Gem + platinum & 1.11 & $0.11(0.07)$ \\
\hline JMIL & Peme + platinum & Gem + platinum & 0.95 & $-0.05(0.13)$ \\
\hline JO19907 & $\mathrm{Pac} / \mathrm{nab}-\mathrm{pac}+$ platinum & $\mathrm{Pac}+$ bev + platinum & 0.61 & $-0.49(0.19)$ \\
\hline KEYNOTE-021G & Peme + platinum & Pembro + peme + platinum & 0.53 & $-0.63(0.24)$ \\
\hline KEYNOTE-189 & Peme + platinum & Pembro + peme + platinum & 0.52 & $-0.65(0.10)$ \\
\hline NAVotrial01 & Peme + platinum & Vin + platinum & 1.16 & $0.15(0.19)$ \\
\hline PointBreak & $\mathrm{Pac}+$ bev + platinum & Peme + bev + platinum & 0.83 & $-0.19(0.08)$ \\
\hline PRONOUNCE & Peme + platinum & $\mathrm{Pac}+\mathrm{bev}+$ platinum & 0.94 & $-0.06(0.12)$ \\
\hline Rodrigues-Pereira (2011) & Peme + platinum & Doc + platinum & 1.04 & $0.04(0.15)$ \\
\hline Sun (2015) & Peme + platinum & Gem + platinum & 1.33 & $0.29(0.12)$ \\
\hline TRAIL & Peme + platinum & Doc + platinum & 0.97 & $-0.03(0.16)$ \\
\hline
\end{tabular}

Atezo: Atezolizumab; Bev: Bevacizumab; Doc: Docetaxel; Gem: Gemcitabine; HR: Hazard ratio; Pac: Paclitaxel; Pembro: Pembrolizumab; Peme: Pemetrexed; SE: Standard error; Vin: Vinorelbine.

Notably, trials differed in the proportion of east Asian patients enrolled. Although it is considered a positive prognostic factor in metastatic NSCLC, east Asian ethnicity is not considered a treatment-effect modifier [86]. In addition, there was variation among trials in the proportion of males enrolled. Female sex is associated with improved chemotherapy outcomes in nonsquamous NSCLC [87-89]. Although KEYNOTE-021G had a higher proportion of female patients than any other trial, sex was balanced between the study arms and, consequently, female sex is unlikely to modify the treatment effect in this trial. Imbalances in sex between study arms in Rodrigues-Pereira 2011 and Johnson 2004 may have caused the efficacy of docetaxel + platinum and paclitaxel + bevacizumab + platinum to be overestimated [6].

Five trials permitted within trial crossover following disease progression, potentially biasing the NMA results for OS. In KEYNOTE-021G and KEYNOTE-189, patients could crossover from pemetrexed + platinum to pembrolizumab monotherapy [6,7]. As a result, the efficacy of pembrolizumab + pemetrexed + platinum relative to comparators in the network may be underestimated. Patients randomized to the paclitaxel/nab-paclitaxel + platinum arm could crossover to the paclitaxel/nab-paclitaxel + atezolizumab + platinum arm in IMpower130 protocol versions 1-4 [10]. In Johnson 2004, patients who received platinum + paclitaxel/nab-paclitaxel could crossover to receive bevacizumab monotherapy [58]. This may cause the efficacy of platinum + paclitaxel/nabpaclitaxel relative to alternative interventions to be overestimated. In TRAIL, patients on either arm could crossover to receive the other study drug or an EGFR inhibitor []. Because the proportion of patients who crossed over was similar in each arm, crossover was unlikely to bias the estimate of docetaxel + platinum's treatment effect relative to other interventions in the network.

The model relied on the assumptions of random effects and proportional hazards. In networks for both OS and PFS, there were enough trials along each connection to employ the random-effects model reliably. Because the random-effects model assumes that each study has its own true treatment effect and these are assumed to follow a distribution around an overall mean (the meta-analysis mean), the model is more plausible than fixed effects because it is consistent with the presence of effect modifiers. Additionally, the analysis was conducted using a proportional hazards model, which rests on the assumption that the hazard functions of competing interventions for OS and PFS do not cross. Finally, this study did not make comparisons of regimens by PD-L1 expression level due to differences in PD-L1 assays, scoring and cutoff points employed in trials conducted by different study sponsors [90,91]. Comparisons of regimens in PD-L1-expressing patients are an important area for future research. 


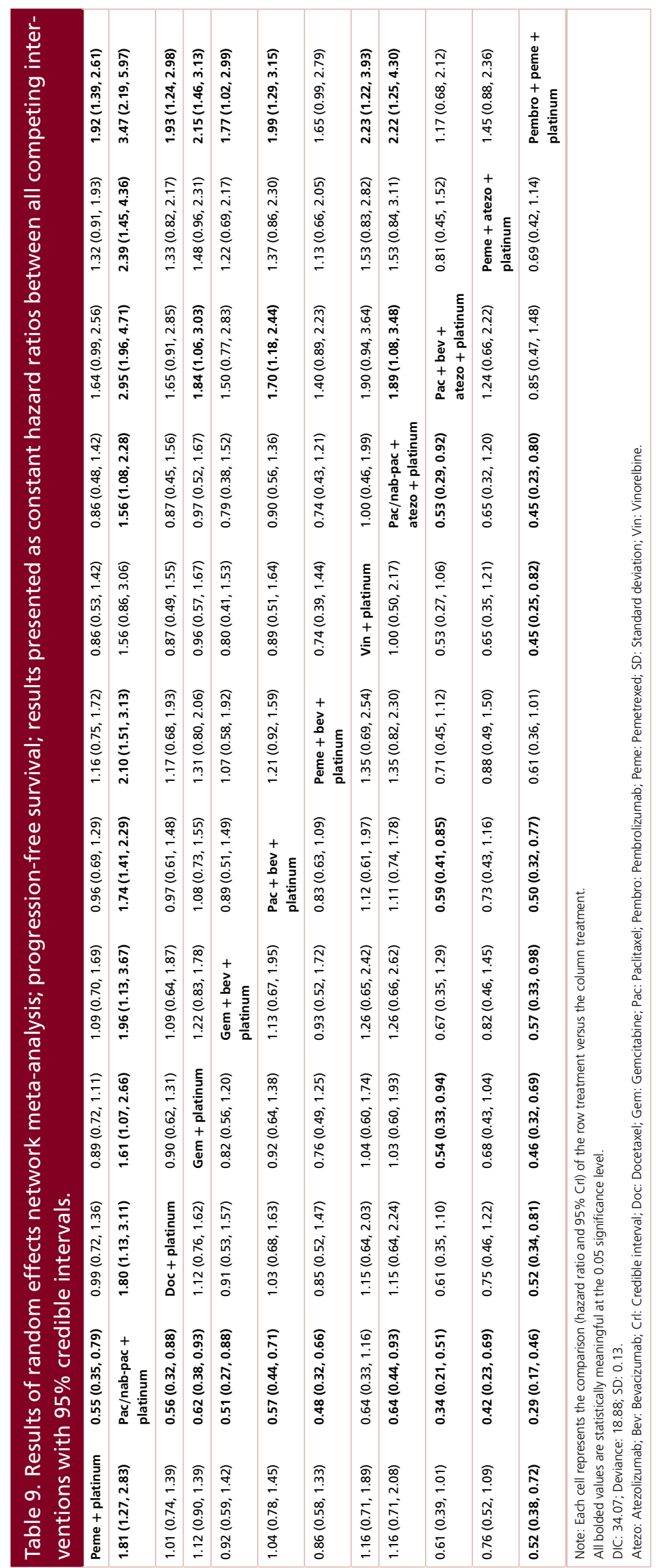




\section{Table 10. Ranking of treatments, overall survival and random effects.}

\begin{tabular}{|c|c|}
\hline Treatment & Probability of being the best \\
\hline Pembro + peme + platinum & 0.956188 \\
\hline $\mathrm{Pac}+\mathrm{bev}+$ atezo + platinum & 0.025688 \\
\hline Peme + atezo + platinum & 0.00675 \\
\hline Vin + platinum & 0.005625 \\
\hline Doc + platinum & 0.003875 \\
\hline $\mathrm{Pac} /$ nab-pac + atezo + platinum & 0.001188 \\
\hline Peme + bev + platinum & 0.0005 \\
\hline Gem + bev + platinum & 0.000188 \\
\hline Peme + platinum & 0 \\
\hline $\mathrm{Pac} /$ nab-pac + platinum & 0 \\
\hline Gem + platinum & 0 \\
\hline $\mathrm{Pac}+\mathrm{bev}+$ platinum & 0 \\
\hline
\end{tabular}

\section{Table 11. Ranking of treatments, progression-free survival, random effects.}

\section{Treatment}

Pembro + peme + platinum

$\mathrm{Pac}+\mathrm{bev}+$ atezo + platinum

Peme + atezo + platinum

Peme + bev + platinum

Gem + bev + platinum

Vin + platinum

$\mathrm{Pac} /$ nab-pac + atezo + platinum

Doc + platinum

Gem + platinum

Peme + platinum

$\mathrm{Pac} /$ nab-pac + platinum

$\mathrm{Pac}+\mathrm{bev}+$ platinum

Probability of being the best
0.672750
0.241313
0.045313
0.016188
0.015625
0.004063
0.002875
0.001750
0.000125
0
0
0

Atezo: Atezolizumab; Bev: Bevacizumab; Doc: Docetaxel; Gem: Gemcitabine; Pac: Paclitaxel; Pembro: Pembrolizumab; Peme: Pemetrexed; Vin: Vinorelbine.

Of all lung cancer diagnoses, NSCLC accounts for $80-85 \%$ of cases and has poor survival rates among late-stage NSCLC [92]. In the USA, the 5-year survival rate is 26,10 and $<1 \%$ for stage IIIB, stage IVA and stage IVB NSCLC, respectively [92]. This analysis reflects the OS and PFS benefit provided by the pembrolizumab + pemetrexed + platinum regimen for those diagnosed with metastatic, nonsquamous NSCLC. In conclusion, pembrolizumab + pemetrexed + platinum had a better HR for survival compared with alternative interventions. This benefit was statistically significant versus all platinum doublets, platinum doublet + bevacizumab, platinum $+\mathrm{paclitaxel} / \mathrm{nab}-$ paclitaxel + atezolizumab and pemetrexed + atezolizumab + platinum for the first-line treatment of metastatic nonsquamous NSCLC, with respect to OS. There was a numerical benefit for OS compared with paclitaxel + bevacizumab + atezolizumab + platinum. Additionally, pembrolizumab + pemetrexed + platinum had a statistically significant benefit for PFS over all platinum doublet therapies, as well as paclitaxel + bevacizumab + platinum, gemcitabine + bevacizumab + platinum and paclitaxel/nab-paclitaxel + atezolizumab + platinum.

\section{Future perspective}

Providing significant increases in survival, PD-1/PD-L1-directed therapies are an important innovation in the firstline treatment of nonsquamous NSCLC. The accumulation of clinical experience with PD-1/PD-L1 inhibitors in this population over the next 5-10 years will provide opportunities to incorporate new clinical trials as well as real-world evidence into future analyses in order to verify these conclusions. 


\section{Executive summary}

Background

- Several clinical trials evaluating PD-1/PD-L1 inhibitors in combination with traditional chemotherapy regimens for first-line treatment of nonsquamous non-small-cell lung cancer (NSCLC) have been recently published.

- In order to evaluate the relative efficacy of treatments that have not been studied head-to-head in this population, a systematic literature review and network meta-analysis were conducted in accordance with a prespecified study protocol.

Systematic literature review

- The systematic review, current through November 2018, was used to identify studies evaluating approved first-line treatments in metastatic nonsquamous NSCLC patients without known EGFR mutations or ALK translocations.

- Fifty-five publications pertaining to 22 trials formed a connected network with 12 interventions (total intent to treat population: 10,826).

Bayesian network meta-analysis methodology

- Bayesian network meta-analysis allows for simultaneous comparison of all included interventions and estimates relative treatment effects between any pair of interventions for a connected network.

- With Bayesian methodology, it is possible to calculate the probability of being the best treatment out of all other interventions included in a connected network.

Feasibility assessment

- The included trials were similar in the distribution of trial characteristics and patient characteristics including Eastern Cooperative Oncology Group performance status, disease stage, and sex distribution.

- Substantial variation in ethnicity was observed between trials. Six trials were conducted exclusively in east Asian patients and five trials reported that fewer than $15 \%$ of enrolled patients were east Asian. Although east Asian ethnicity is a prognostic factor for patients with nonsquamous NSCLC, it is not thought to be a relative treatment-effect modifier.

Network meta-analysis results

- This analysis suggests combining pembrolizumab with pemetrexed and platinum results in longer overall survival compared with all platinum doublet chemotherapy regimens as well as platinum doublets combined with atezolizumab or bevacizumab for the first-line treatment of metastatic nonsquamous NSCLC.

- Based on median rankings and probabilities of being the best treatment, pembrolizumab + pemetrexed + platinum had a median rank of 1 and has the highest probability of being the best treatment regimen for both overall and progression-free survivals.

Financial \& competing interests disclosure

The research was funded by Merck \& Co., Inc. Authors R Insinga, A Arunachalam and TA Burke are employees of Merck \& Co., Inc. AM Frederickson, S Arndorfer, I Zhang and M Lorenzi are employees of Precision Xtract, a consultancy contracted by Merck \& Co., Inc. to conduct research. GR Simon is a paid consultant to Merck \& Co., Inc. The authors have no other relevant affiliations or financial involvement with any organization or entity with a financial interest in or financial conflict with the subject matter or materials discussed in the manuscript apart from those disclosed.

\section{Open access}

This work is licensed under the Attribution-NonCommercial-NoDerivatives 4.0 Unported License. To view a copy of this license, visit http://creativecommons.org/licenses/by-nc-nd/4.0/

\section{References}

Papers of special note have been highlighted as: $\bullet$ of interest; $\bullet \bullet$ of considerable interest

1. Herbst RS, Baas P, Kim DW et al. Pembrolizumab versus docetaxel for previously treated, PD-L1-positive, advanced non-small-cell lung cancer (KEYNOTE-010): a randomised controlled trial. Lancet 387(10027), 1540-1550 (2016).

2. Borghaei H, Paz-Ares L, Horn L et al. Nivolumab versus docetaxel in advanced nonsquamous non-small-cell lung cancer. N. Engl. J. Med. 373(17), 1627-1639 (2015).

3. Brahmer J, Reckamp KL, Baas P et al. Nivolumab versus docetaxel in advanced squamous-cell non-small-cell lung cancer. N. Engl. J. Med. 373(2), 123-135 (2015).

4. Rittmeyer A, Barlesi F, Waterkamp D et al. Atezolizumab versus docetaxel in patients with previously treated non-small-cell lung cancer (OAK): a Phase III, open-label, multicentre randomised controlled trial. Lancet 389(10066), 255-265 (2017). 
5. Reck M, Rodriguez-Abreu D, Robinson AG et al. Pembrolizumab versus chemotherapy for PD-L1-positive non-small-cell lung cancer. N. Engl. J. Med. 375(19), 1823-1833 (2016).

6. Langer CJ, Gadgeel SM, Borghaei $\mathrm{H}$ et al. Carboplatin and pemetrexed with or without pembrolizumab for advanced, non-squamous non-small-cell lung cancer: a randomised, Phase II cohort of the open-label KEYNOTE-021 study. Lancet Oncol. 17(11), 1497-1508 (2016).

-. Pembrolizumab in combination with platinum doublet chemotherapy followed by pemetrexed maintenance provided chemotherapy-naive patients with significantly improved progression-free survival and objective response compared with standard platinum-based chemotherapy.

7. Gandhi L, Rodriguez-Abreu D, Gadgeel S et al. Pembrolizumab plus chemotherapy in metastatic non-small-cell lung cancer. N. Engl. J. Med. 378(22), 2078-2092 (2018).

-. Pembrolizumab in addition to pemetrexed and platinum chemotherapy followed by pemetrexed maintenance offered chemotherapy-naive nonsquamous non-small-cell lung cancer (NSCLC) patients without EGFR and anaplastic lymphoma kinase (ALK) mutations statistically improved overall and progression-free survival compared with standard platinum doublet chemotherapy.

8. Socinski MA, Jotte RM, Cappuzzo F et al. Atezolizumab for first-line treatment of metastatic nonsquamous NSCLC. N. Engl. J. Med. 378(24), 2288-2301 (2018).

-. Atezolizumab in combination with bevacizumab and platinum chemotherapy significantly improved overall and progression-free survivals in patients with chemotherapy-naive metastatic nonsquamous NSCLC without EGFR or ALK genetic mutations compared with standard platinum doublet + bevacizumab chemotherapy.

9. Socinski MA, Jotte RM, Cappuzzo F et al. Overall survival (OS) analysis of IMpower150, a randomized Ph 3 study of atezolizumab (atezo)+chemotherapy (chemo) \pm bevacizumab (bev) vs chemo+bev in 1L nonsquamous (NSQ) NSCLC. J. Clin. Oncol. 36, 9002 (2018).

10. Cappuzzo F, Mccleod M, Hussein M et al. LBA53 IMpower130: progression-free survival (PFS) and safety analysis from a randomised Phase III study of carboplatin + nab-paclitaxel (CnP) with or without atezolizumab (atezo) as first-line (1L) therapy in advanced non-squamous NSCLC. Ann. Oncol. 29(suppl_8), mdy424.065-mdy424.065 (2018).

-. Atezolizumab with paclitaxel/nab-paclitaxel and platinum offered improved overall and progression-free survivals compared with standard platinum-doublet chemotherapy in advanced nonsquamous NSCLC patients without EGFR or ALK mutations.

11. Papadimitrakopoulou VEA. IMpower132: PFS and safety results with $1 \mathrm{~L}$ atezolizumab + carboplatin/cisplatin + pemetrexed in stage IV non-squamous NSCLC. 13, S332-S333 (2018).

-• Atezolizumab in combination with pemetrexed and platinum followed by atezolizumab + pemetrexed maintenance therapy offered improved progression-free survival compared with pemetrexed + platinum chemotherapy in chemotherapy-naive patients with stage IV nonsquamous NSCLC.

12. NCCN clinical practice guidelines in oncology: non-small cell lung cancer (Version 6.2018). (2018). www.nccn.org/professionals/physician_gls/PDF/nscl.pdf

13. Planchard D, Popat S, Kerr K et al. Metastatic non-small cell lung cancer: ESMO Clinical Practice Guidelines for diagnosis, treatment and follow-up†. Ann. Oncol 29(Supplement_4), iv192-iv237 (2018).

14. Ades AE. A chain of evidence with mixed comparisons: models for multi-parameter synthesis and consistency of evidence. Stat. Med. 22(19), 2995-3016 (2003).

15. Caldwell DM, Ades AE, Higgins JPT. Simultaneous comparison of multiple treatments: combining direct and indirect evidence. BMJ 331(7521), 897-900 (2005).

16. Lu G, Ades AE. Combination of direct and indirect evidence in mixed treatment comparisons. Stat. Med. 23(20), 3105-3124 (2004).

17. Higgins JPT, Altman DG, Gøtzsche PC et al. The Cochrane Collaboration's tool for assessing risk of bias in randomised trials. BMJ 343 , d5928 (2011).

18. Dias S, Welton NJ, Sutton AJ, Ades A. NICE DSU technical support document 2: a generalized linear modeling framework for pairwise and network meta-analysis of randomized controlled trials. (last updated September 2016), (2011). http://nicedsu.org.uk/wp-content/uploads/2017/05/TSD2-General-meta-analysis-corrected-2Sep2016v2.pdf

19. Bucher HC, Guyatt GH, Griffith LE, Walter SD. The results of direct and indirect treatment comparisons in meta-analysis of randomized controlled trials. J. Clin. Epidemiol. 50(6), 683-691 (1997).

20. Li L, Catala-Lopez F, Alonso-Arroyo A et al. The global research collaboration of network meta-analysis: a social network analysis. PLoS ONE 11(9), e0163239 (2016).

21. Laws A, Kendall R, Hawkins N. A comparison of national guidelines for network meta-analysis. Value Health 17(5), 642-654 (2014).

22. Cope S, Zhang J, Saletan S, Smiechowski B, Jansen JP, Schmid P. A process for assessing the feasibility of a network meta-analysis: a case study of everolimus in combination with hormonal therapy versus chemotherapy for advanced breast cancer. BMC Med. 12(1), 1-17 (2014). 
- A step-wise framework for the feasibility assessment prior to conducting a network meta-analysis provides transparency regarding heterogeneity of the evidence base and clarifies if underlying assumptions are outlined along with risks or benefits associated with these assumptions.

23. Cope S, Jansen JP. Quantitative summaries of treatment effect estimates obtained with network meta-analysis of survival curves to inform decision-making. BMC Med. Res. Methodol. 13, 147 (2013).

- Under the Bayesian framework, network meta-analyses can provide rank probabilities of treatment effects to help better inform decision making on which treatment is most likely the best for the population assessed in the connected network of interest.

24. Guyot P, Ades AE, Ouwens MJ, Welton NJ. Enhanced secondary analysis of survival data: reconstructing the data from published Kaplan-Meier survival curves. BMC Med. Res. Methodol. 12, 9 (2012).

25. Rstudio Team. (2015). RStudio: Integrated Development for R. RStudio, Inc., Boston, MA, USA www.rstudio.com/

26. Reck M, Pawel JV, Zatloukal P et al. Phase III trial of cisplatin plus gemcitabine with either placebo or bevacizumab as first-line therapy for nonsquamous non-small-cell lung cancer: AVAiL. J. Clin. Oncol. 27(8), 1227-1234 (2009).

27. Reck M, Von Pawel J, Zatloukal P et al. Overall survival with cisplatin-gemcitabine and bevacizumab or placebo as first-line therapy for nonsquamous non-small-cell lung cancer: results from a randomised Phase III trial (AVAiL). Ann. Oncol. 21(9), 1804-1809 (2010).

28. Mok TS, Hsia T-C, Tsai C-M et al. Efficacy of bevacizumab with cisplatin and gemcitabine in Asian patients with advanced or recurrent non-squamous non-small cell lung cancer who have not received prior chemotherapy: a substudy of the Avastin in lung trial. Asia Pac. J. Clin. Oncol. 7(s2), 4-12 (2011).

29. Zhou C, Wu YL, Chen G et al. BEYOND: a randomized, double-blind, placebo-controlled, multicenter, Phase III study of first-line carboplatin/paclitaxel plus bevacizumab or placebo in Chinese patients with advanced or recurrent nonsquamous non-small-cell lung cancer. J. Clin. Oncol. 33(19), 2197-2204 (2015).

30. Carbone DP, Reck M, Paz-Ares L et al. First-line nivolumab in stage IV or recurrent non-small-cell lung cancer. N. Engl. J. Med. 376(25), 2415-2426 (2017).

31. Socinski M, Creelan B, Horn L et al. PR CheckMate 026: a Phase III trial of nivolumab vs investigator's choice (IC) of platinum-based doublet chemotherapy (PT-DC) as first-line therapy for stage IV/recurrent programmed death ligand 1 (PD-L1)-positive NSCLC. Ann. Oncol.doi:10.1093/annonc/mdw435 (2016) (Epub ahead of print).

32. Koyama R, Udagawa H, Sugiyama E et al. 1436P Randomized Phase II study comparing cisplatin + pemetrexed + bevacizumab with carboplatin + paclitaxel + bevacizumab in treatment-naive advanced non-squamous non-small-cell lung cancer (CLEAR study). Ann. Oncol. 29(suppl_8), mdy292.058-mdy292.058 (2018).

33. Atagi S, Udagawa H, Sugiyama E et al. Randomized Phase II study comparing CBDCA+PTX+BEV and CDDP+PEM+BEV in treatment-naive advanced non-sq NSCLC (clear study). J. Thoracic Oncol. 12 (11 Supplement 2), S1961 (2017).

34. Harada T, Udagawa H, Sugiyama E et al. Randomized Phase II study comparing CBDCA+PTX+BEV and CDDP+PEM+BEV in treatment-naive advanced non-Sq NSCLC (CLEAR study). J. Thoracic Oncol. 13(10 Supplement), S472-S473 (2018).

35. Sandler A, Gray R, Perry MC et al. Paclitaxel-carboplatin alone or with bevacizumab for non-small-cell lung cancer. N. Engl. J. Med. 355(24), 2542-2550 (2006).

36. Sandler A, Yi J, Dahlberg S et al. Treatment outcomes by tumor histology in Eastern Cooperative Group Study E4599 of bevacizumab with paclitaxel/carboplatin for advanced non-small-cell lung cancer. J. Thoracic Oncol. 5(9), 1416-1423 (2010).

37. Tyagi P, Belani CP. Bevacizumab, when added to paclitaxel/carboplatin, prolongs survival in previously untreated patients with advanced non-small-cell lung cancer: preliminary results from the ECOG 4599 trial. Clin. Lung Cancer 6(5), 276-278 (2005).

38. Gerber DE, Dahlberg SE, Sandler AB et al. Baseline tumour measurements predict survival in advanced non-small-cell lung cancer. Br. J. Cancer 109(6), 1476-1481 (2013).

39. Galetta D, Cinieri S, Pisconti $S$ et al. Cisplatin/pemetrexed followed by maintenance pemetrexed versus carboplatin/paclitaxel/bevacizumab followed by maintenance bevacizumab in advanced nonsquamous lung cancer: the GOIM (Gruppo Oncologico Italia Meridionale) ERACLE Phase III randomized trial. Clin. Lung Cancer 16(4), 262-273 (2015).

40. Gronberg BH, Bremnes RM, Flotten O et al. Phase III study by the norwegian lung cancer study group: pemetrexed plus carboplatin compared with gemcitabine plus carboplatin as first-line chemotherapy in advanced non-small-cell lung cancer. J. Clin. Oncol. 27(19), 3217-3224 (2009).

41. Brown T, Pilkington G, Bagust A et al. Clinical effectiveness and cost-effectiveness of first-line chemotherapy for adult patients with locally advanced or metastatic non-small-cell lung cancer: a systematic review and economic evaluation. Health Technol. Assess. 17(31), 1-278 (2013).

42. Socinski M. Overall survival (OS) analysis of IMpower150, a randomized Ph 3 study of atezolizumab (atezo)+chemotherapy (chemo) \pm bevacizumab (bev) vs chemo+bev in $1 \mathrm{~L}$ nonsquamous (NSQ) NSCLC. ASCO Annu. Meeting 36(15_suppl), 9002 (2018).

43. Reck M. Patient-reported outcomes (PROs) in the randomized, Phase III IMpower150 study of atezolizumab (atezo)+chemotherapy (chemo) \pm bevacizumab (bev) vs chemo+bev in 1L nonsquamous metastatic NSCLC (mNSCLC). ASCO Annu. Meeting 36(15_suppl.), 9047 (2018). 
44. Reck M, Socinski MA, Cappuzzo F et al. LBA1_PR primary PFS and safety analyses of a randomized Phase III study of carboplatin + paclitaxel +/- bevacizumab, with or without atezolizumab in 1L non-squamous metastatic NSCLC (IMpower150). Ann. Oncol. 28(Supplement 11), xi31 (2017).

45. Kowanetz M, Socinski M, Zou W et al. IMpower150: efficacy of atezolizumab plus bevacizumab and chemotherapy in 1L metastatic nonsquamous NSCLC across key subgroups. Presented at: 2018 AACR Annual Meeting. Chicago, IL, USA. Abstract CT076, 14-18 April 2018.

46. Reck M, Socinski MA, Cappuzzo F et al. Primary PFS and safety analyses of a randomized Phase III study of carboplatin + paclitaxel +/- bevacizumab, with or without atezolizumab in 1L non-squamous metastatic NSCLC (IMpower150). Ann. Oncol. 28(Supplement 11), xi31 (2017).

47. Reck M, Socinski M, Cappuzzo F et al. Primary PFS and safety analyses of a randomized Phase III study of carboplatin + paclitaxel +/bevacizumab, with or without atezolizumab in 1L non-squamous metastatic NSCLC (IMpower150). Oncol. Res. Treat. 41(Supplement 1), 187 (2018).

48. Rothenstein J, Spira A, Velcheti V et al. IMpower150: impact of chemotherapy cycles in 1L metastatic NSCLC in patients treated with atezolizumab + bevacizumab. J. Thoracic Oncol. 13 (10 Supplement), S495 (2018).

49. Reck M, Wehler T, Orlandi F et al. 1386PDIMpower150: clinical safety, tolerability and immune-related adverse events in a Phase III study of atezolizumab (atezo) + chemotherapy (chemo) \pm bevacizumab (bev) vs chemo + bev in $1 \mathrm{~L}$ nonsquamous NSCLC. Ann. Oncol. 29(suppl._8), mdy292.009-mdy292.009 (2018).

50. Jang J, Kim HK, Cho BC et al. Randomized Phase II study comparing weekly docetaxel-cisplatin vs gemcitabine-cisplatin in elderly or poor performance status patients with advanced non-small-cell lung cancer. Cancer Chemother. Pharmacol. (2017).

51. Scagliotti GV, Parikh P, Von Pawel J et al. Phase III study comparing cisplatin plus gemcitabine with cisplatin plus pemetrexed in chemotherapy-naive patients with advanced-stage non-small-cell lung cancer. J. Clin. Oncol. 26(21), 3543-3551 (2008).

52. Novello S, Pimentel FL, Douillard JY et al. Safety and resource utilization by non-small-cell lung cancer histology: results from the randomized Phase III study of pemetrexed plus cisplatin versus gemcitabine plus cisplatin in chemonaive patients with advanced non-small-cell lung cancer. J. Thoracic Oncol. 5(10), 1602-1608 (2010).

53. Syrigos KN, Vansteenkiste J, Parikh P et al. Prognostic and predictive factors in a randomized Phase III trial comparing cisplatin-pemetrexed versus cisplatin-gemcitabine in advanced non-small-cell lung cancer. Ann. Oncol. 21(3), 556-561 (2010).

54. Yang CH, Simms L, Park K, Lee JS, Scagliotti G, Orlando M. Efficacy and safety of cisplatin/pemetrexed versus cisplatin/gemcitabine as first-line treatment in east Asian patients with advanced non-small-cell lung cancer: results of an exploratory subgroup analysis of a Phase III trial. J. Thoracic Oncol. 5(5), 688-695 (2010).

55. Wu Y-L, Lu S, Cheng Y et al. Efficacy and safety of pemetrexed/cisplatin versus gemcitabine/cisplatin as first-line treatment in Chinese patients with advanced nonsquamous non-small-cell lung cancer. Lung Cancer 85(3), 401-407 (2014).

56. Eli Lilly and Company. A study comparing two different chemotherapy types in Chinese patients with advanced non-small-cell lung cancer. In: ClinicalTrials.gov. National Library of Medicine (USA). Bethesda, MD, USA, 28 September 2016, (2013). https://clinicaltrials.gov/ct2/show/NCT01005680

57. Niho S, Kunitoh H, Nokihara $\mathrm{H}$ et al. Randomized Phase II study of first-line carboplatin-paclitaxel with or without bevacizumab in Japanese patients with advanced non-squamous non-small-cell lung cancer. Lung Cancer 76(3), 362-367 (2012).

58. Johnson DH, Fehrenbacher L, Novotny WF et al. Randomized Phase II trial comparing bevacizumab plus carboplatin and paclitaxel with carboplatin and paclitaxel alone in previously untreated locally advanced or metastatic non-small-cell lung cancer. J. Clin. Oncol. 22(11), 2184-2191 (2004).

59. Langer C, Gaddgeel SM, Borghaei H et al. Randomized, Phase II study of carboplatin and pemetrexed with or without pembrolizumab as first-line therapy for advanced NSCLC: KEYNOTE-021 cohort G. Ann. Oncol. Conference 27, vi552-vi587 (2016).

60. Langer C, Gadgeel S, Borghaei H et al. MA09.02 Pembrolizumab + carboplatin and pemetrexed as 1st-line therapy for advanced non-small-cell lung cancer: KEYNOTE-021 cohort G. J. Thoracic Oncol. 12(1), S391-S392 (2017).

61. Papadimitrakopoulou V, Gadgeel SM, Borghaei $\mathrm{H}$ et al. First-line carboplatin and pemetrexed (CP) with or without pembrolizumab (pembro) for advanced nonsquamous NSCLC: updated results of KEYNOTE-021 cohort G. J. Clin. Oncol. 35(15_suppl), 9094-9094 (2017).

62. Borghaei $\mathrm{H}$, Langer $\mathrm{C}$, Gadgeel $\mathrm{S}$ et al. Pemetrexed-carboplatin plus pembrolizumab as first-line therapy for advanced nonsquamous NSCLC: KEYNOTE-021 cohort G update. J. Thoracic Oncol. 12(11 Supplement 2), S1791 (2017).

63. Borghaei H, Langer CJ, Gadgeel S et al. Updated results from KEYNOTE-021 cohort G: a randomized, Phase II study of pemetrexed and carboplatin (PC) with or without pembrolizumab (pembro) as first-line therapy for advanced nonsquamous NSCLC. Ann. Oncol.28(Supplement 5), v636-v637 (2017).

64. Borghaei H, Langer CJ, Gadgeel S et al. 24-Month overall survival from KEYNOTE-021 cohort G: pemetrexed and carboplatin with or without pembrolizumab as first-line therapy for advanced nonsquamous non-small-cell lung cancer. J. Thoracic Oncol. 18(30942), S1556-0864 (2018). 
65. Gentzler R. 24-month overall survival from KEYNOTE-021 cohort G: pemetrexed-carboplatin plus pembrolizumab as first-line therapy for advanced nonsquamous NSCLC. ASCO Annual Meeting 36(15_suppl), 9026 (2018).

66. Reck M, Rodriguez-Abreu D, Robinson A et al. KEYNOTE-024: pembrolizumab (pembro) vs platinum-based chemotherapy (chemo) as first-line therapy for advanced NSCLC with a PD-L1 tumor proportion score (TPS) $>=50 \%$. Ann. Oncol. 27(Supplement 9), ix139 (2016).

67. Brahmer J, Rodriguez-Abreu D, Robinson A et al. Updated analysis of keynote-024: pembrolizumab vs platinum-based chemotherapy for advanced NSCLC with PD-L1 TPS > = 50\%. J. Thoracic Oncol. Conference: 18 th world conference on lung cancer of the international association for the study of lung cancer, IASLC. 2017. Japan, 12(11 Supplement 2), S1793-S1794 (2017).

68. Brahmer JR, Rodriguez-Abreu D, Robinson AG et al. Health-related quality-of-life results for pembrolizumab versus chemotherapy in advanced, PD-L1-positive NSCLC (KEYNOTE-024): a multicentre, international, randomised, open-label Phase III trial. Lancet Oncol. (pagination), 18(12), 1600-1609 (2017).

69. Reck M, Rodríguez-Abreu D, Robinson AG et al. Treatment switching-adjusted overall survival (OS) in KEYNOTE-024: first-line pembrolizumab versus chemotherapy in patients with advanced non-small cell lung cancer (NSCLC). J. Thoracic Oncol. Conference 8th European Lung Cancer Congress, ELCC 2018. Switzerland. 2013 13(2014 Supplement 2011), S2112-S2113 (2018).

70. Gandhi L. CT075- KEYNOTE-189: randomized, double-blind, Phase III study of pembrolizumab (pembro) or placebo plus pemetrexed (pem) and platinum as first-line therapy for metastatic NSCLC. Presented at: 2018 AACR Annual Meeting McCormick Place North/South Chicago, IL, USA 14-18 April (2018).

71. Garassino MC, Rodriguez-Abreu D, Gadgeel SM et al. Health-related quality of life (HRQoL) in the KEYNOTE-189 study of pembrolizumab (pembro) or placebo (pbo)+pemetrexed (pem)+platinum (plt) for metastatic NSCLC. J. Clin. Oncol. 36(15_suppl.), 9021 (2018).

72. Hui R, Garassino MC, Rodriguez-Abreu D et al. Health-related quality of life (HRQoL) with pembrolizumab or placebo + pemetrexed + platinum in patients with metastatic non-squamous NSCLC: Keynote-189. Asia Pac. J. Clin. Oncol. 14(Supplement 3), 47 (2018).

73. Rodriguez Abreu D, Garassino MC, Esteban E et al. KEYNOTE-189 study of pembrolizumab (pembro) plus pemetrexed (pem) and platinum vs placebo plus pem and platinum for untreated, metastatic, nonsquamous NSCLC: does choice of platinum affect outcomes? Ann. Oncol. 29(suppl_8), mdy292.086-mdy292.086 (2018).

74. Kristensen A, Solheim TS, Amundsen T et al. Measurement of health-related quality of life during chemotherapy - the importance of timing. Acta Oncol. (2017).

75. Bennouna J, Havel L, Krzakowski $M$ et al. Oral vinorelbine plus cisplatin as first-line chemotherapy in nonsquamous non-small-cell lung cancer: final results of an international randomized Phase II study (NAVotrial 01). Clin. Lung Cancer 15(4), 258-265 (2014).

76. Patel JD, Socinski MA, Garon EB et al. PointBreak: a randomized Phase III study of pemetrexed plus carboplatin and bevacizumab followed by maintenance pemetrexed and bevacizumab versus paclitaxel plus carboplatin and bevacizumab followed by maintenance bevacizumab in patients with stage IIIB or IV nonsquamous non-small-cell lung cancer. J. Clin. Oncol. 31(34), 4349-4357 (2013).

77. Spigel DR, Patel JD, Reynolds CH et al. Quality of life analyses from the randomized, open-label, Phase III PointBreak study of pemetrexed-carboplatin-bevacizumab followed by maintenance pemetrexed-bevacizumab versus paclitaxel-carboplatin-bevacizumab followed by maintenance bevacizumab in patients with stage IIIB or IV nonsquamous non-small-cell lung cancer. J. Clin. Oncol. 10(2), 353-359 (2015).

78. Reynolds CH, Patel JD, Garon EB et al. Exploratory subset analysis of African Americans from the PointBreak study: pemetrexed-carboplatin-bevacizumab followed by maintenance pemetrexed-bevacizumab versus paclitaxel-carboplatin-bevacizumab followed by maintenance bevacizumab in patients with stage IIIB/IV nonsquamous non-small-cell lung cancer. Clin. Lung Cancer 16(3), 200-208 (2015).

79. Zinner RG, Obasaju CK, Spigel DR et al. PRONOUNCE: randomized, open-label, Phase III study of first-line pemetrexed + carboplatin followed by maintenance pemetrexed versus paclitaxel + carboplatin + bevacizumab followed by maintenance bevacizumab in patients ith advanced nonsquamous non-small-cell lung cancer. J. Thoracic Oncol. 10(1), 134-142 (2015).

80. Rodrigues-Pereira J, Kim JH, Magallanes M et al. A randomized Phase III trial comparing pemetrexed/carboplatin and docetaxel/carboplatin as first-line treatment for advanced, nonsquamous non-small-cell lung cancer. J. Thoracic Oncol. 6(11), 1907-1914 (2011).

81. Spigel DR, Hainsworth JD, Shipley DL et al. A randomized Phase II trial of pemetrexed/gemcitabine/bevacizumab or pemetrexed/carboplatin/bevacizumab in the first-line treatment of elderly patients with advanced non-small-cell lung cancer. J. Thorac. Oncol. 7(1), 196-202 (2012).

82. Sun JM, Ahn JS, Jung SH et al. Pemetrexed plus cisplatin versus gemcitabine plus cisplatin according to thymidylate synthase expression in nonsquamous non-small-cell lung cancer: a biomarker-stratified randomized Phase II trial. J. Clin. Oncol. 33(22), 2450-2456 (2015).

83. Park CK, Oh IJ, Kim KS et al. Randomized Phase III study of docetaxel plus cisplatin versus pemetrexed plus cisplatin as first-line treatment of nonsquamous non-small-cell lung cancer: a TRAIL trial. Clin. Lung Cancer 08 (2017).

84. Zhang X, Lu J, Xu J et al. Pemetrexed plus platinum or gemcitabine plus platinum for advanced non-small-cell lung cancer: final survival analysis from a multicentre randomized Phase II trial in the east Asia region and a meta-analysis. Respirology 18(1), 131-139 (2013). 
85. Johnson DH, Fehrenbacher L, Novotny WF et al. Randomized Phase II trial comparing bevacizumab plus carboplatin and paclitaxel with carboplatin and paclitaxel alone in previously untreated locally advanced or metastatic non-small-cell lung cancer. J. Clin. Oncol. 22(11), 2184-2191 (2004).

86. Soo RA, Kawaguchi $\mathrm{T}$, Loh $\mathrm{M}$ et al. Differences in outcome and toxicity between Asian and caucasian patients with lung cancer treated with systemic therapy. Future Oncol. 8(4), 451-462 (2012).

87. Wakelee HA, Dahlberg SE, Brahmer JR et al. Differential effect of age on survival in advanced NSCLC in women versus men: analysis of recent Eastern Cooperative Oncology Group (ECOG) studies, with and without bevacizumab. Lung Cancer 76(3), 410-415 (2012).

88. Wheatley-Price P, Blackhall F, Lee SM et al. The influence of sex and histology on outcomes in non-small-cell lung cancer: a pooled analysis of five randomized trials. Ann. Oncol. 21(10), 2023-2028 (2010).

89. Cuyún Carter G, Barrett AM, Kaye JA, Liepa AM, Winfree KB, John WJ. A comprehensive review of nongenetic prognostic and predictive factors influencing the heterogeneity of outcomes in advanced non-small-cell lung cancer. Cancer Manage. Res. 6, 437-449 (2014).

90. Hirsch FR, Mcelhinny A, Stanforth D et al. PD-L1 immunohistochemistry assays for lung cancer: results from Phase I of the blueprint PD-L1 IHC Assay Comparison Project. J. Thoracic Oncol. 12(2), 208-222 (2017).

91. Tsao MS, Kerr KM, Kockx M et al. PD-L1 immunohistochemistry comparability study in real-life clinical samples: results of blueprint Phase II project. J. Thoracic Oncol. 13(9), 1302-1311 (2018).

92. Goldstraw P, Chansky K, Crowley J et al. The IASLC lung cancer staging project: proposals for revision of the TNM stage groupings in the forthcoming (eighth) edition of the TNM classification for lung cancer. J. Thoracic Oncol. 11(1), 39-51 (2016). 